\title{
Oxygen versus air-driven nebulisers for exacerbations of chronic obstructive pulmonary disease: a randomised controlled trial
}

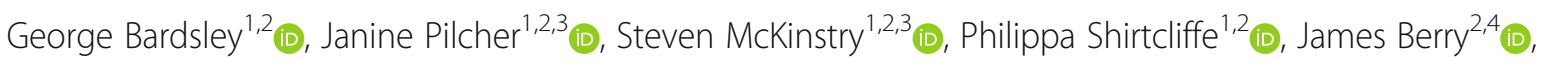
James Fingleton ${ }^{1,2}$, Mark Weatherall ${ }^{4}$ (D) and Richard Beasley ${ }^{1,2,3^{*}}$ (D)

\begin{abstract}
Background: In exacerbations of chronic obstructive pulmonary disease, administration of high concentrations of oxygen may cause hypercapnia and increase mortality compared with oxygen titrated, if required, to achieve an oxygen saturation of 88-92\%. Optimally titrated oxygen regimens require two components: titrated supplemental oxygen to achieve the target oxygen saturation and, if required, bronchodilators delivered by air-driven nebulisation. The effect of repeated air vs oxygen-driven bronchodilator nebulisation in acute exacerbations of chronic obstructive pulmonary disease is unknown. We aimed to compare the effects of air versus oxygen-driven bronchodilator nebulisation on arterial carbon dioxide tension in exacerbations of chronic obstructive pulmonary disease.
\end{abstract}

Methods: A parallel group double-blind randomised controlled trial in 90 hospital in-patients with an acute exacerbation of COPD. Participants were randomised to receive two $2.5 \mathrm{mg}$ salbutamol nebulisers, both driven by air or oxygen at $8 \mathrm{~L} / \mathrm{min}$, each delivered over 15 min with a $5 \mathrm{~min}$ interval in-between. The primary outcome measure was the transcutaneous partial pressure of carbon dioxide at the end of the second nebulisation (35 min). The primary analysis used a mixed linear model with fixed effects of the baseline $\mathrm{PtCO}_{2}$, time, the randomised intervention, and a time by intervention interaction term; to estimate the difference between randomised treatments at 35 min. Analysis was by intention-to-treat.

Results: Oxygen-driven nebulisation was terminated in one participant after $27 \mathrm{~min}$ when the $\mathrm{PtCO}_{2}$ rose by $>10 \mathrm{mmHg}$, a predefined safety criterion. The mean (standard deviation) change in $\mathrm{PtCO}_{2}$ at $35 \mathrm{~min}$ was 3.4 (1.9) $\mathrm{mmHg}$ and 0.1 (1.4) $\mathrm{mmHg}$ in the oxygen and air groups respectively, difference (95\% confidence interval) $3.3 \mathrm{mmHg}(2.7$ to 3.9$), p<0.001$. The proportion of patients with a $\mathrm{PtCO}_{2}$ change $\geq 4 \mathrm{mmHg}$ during the intervention was 18/45 (40\%) and 0/44 (0\%) for oxygen and air groups respectively.

Conclusions: Oxygen-driven nebulisation leads to an increase in $\mathrm{PtCO}_{2}$ in exacerbations of COPD. We propose that airdriven bronchodilator nebulisation is preferable to oxygen-driven nebulisation in exacerbations of COPD.

Trial registration: Australian New Zealand Clinical Trials Registry number ACTRN12615000389505. Registration confirmed on 28/4/15.

Keywords: Air, Bronchodilator agents, Hypercapnia, Nebulisation, Oxygen

\footnotetext{
* Correspondence: richard.beasley@mrinz.ac.nz

${ }^{1}$ Capital and Coast District Health Board, Wellington, New Zealand

${ }^{2}$ Medical Research Institute of New Zealand, Box 7902, Wellington, PO 6242,

New Zealand

Full list of author information is available at the end of the article
}

(c) The Author(s). 2018 Open Access This article is distributed under the terms of the Creative Commons Attribution 4.0 International License (http://creativecommons.org/licenses/by/4.0/), which permits unrestricted use, distribution, and reproduction in any medium, provided you give appropriate credit to the original author(s) and the source, provide a link to the Creative Commons license, and indicate if changes were made. The Creative Commons Public Domain Dedication waiver (http://creativecommons.org/publicdomain/zero/1.0/) applies to the data made available in this article, unless otherwise stated. 


\section{Background}

In acute exacerbations of chronic obstructive pulmonary disease (AECOPD), administration of high concentration oxygen may cause profound hypercapnia and increase mortality, compared with oxygen titrated to achieve an oxygen saturation of between 88 to $92 \%$ [1, 2]. Titrated oxygen regimens require two components: titrated supplemental oxygen to achieve a particular target arterial oxygen saturation measured by pulse oximetry $\left(\mathrm{SpO}_{2}\right)$, and bronchodilators delivered by either air-driven nebulisation or metered-dose inhalers with a spacer. Oxygendriven nebulisation inadvertently exposes patients to high concentrations of inspired oxygen, particularly with prolonged or repeated use as may occur in patients with severe exacerbations during long pre-hospital transfers or if the mask is inadvertently left in place.

We have shown that air-driven bronchodilator nebulisation prevents the increase in arterial partial pressure of carbon dioxide $\left(\mathrm{PaCO}_{2}\right)$ that results from use of oxygen-driven nebulisers in patients with stable COPD [3]. However, there are only two small non-blinded randomised controlled trials of air compared to oxygen-driven nebulisation in patients admitted to hospital with AECOPD [4, 5]. These trials reported that administration of a single bronchodilator dose using oxygen-driven nebulisation increases the $\mathrm{PaCO}_{2}$ in COPD patients who have baseline hypercapnia.

Robust determination of the risks of oxygen-driven nebulisation in AECOPD could identify whether widespread implementation of air-driven nebulisers, or use of metered-dose inhalers through a spacer, are required to ensure safe delivery of bronchodilators to this high-risk patient group. The objective of this study was to compare the effects on $\mathrm{PaCO}_{2}$ of air- and oxygen-driven bronchodilator nebulisation in AECOPD. Our hypothesis was that two doses of oxygen-driven bronchodilator nebulisation would increase the $\mathrm{PaCO}_{2}$ compared with air-driven nebulisation in patients hospitalised with an AECOPD.

\section{Methods}

\section{Trial design and patients}

This was a parallel-group double-blind randomised controlled trial at Wellington Regional Hospital, New Zealand. The full study protocol is available in the online supplement.

Participants were hospital inpatients, $\geq 40$ years of age, with an admission diagnosis of AECOPD. Exclusion criteria included requirement for $\geq 4 \mathrm{~L} / \mathrm{min}$ of oxygen via nasal cannulae to maintain $\mathrm{SpO}_{2}$ between 88 to 92\%; current requirement for non-invasive ventilation (NIV); baseline transcutaneous partial pressure of carbon dioxide $\left(\mathrm{PtCO}_{2}\right)>60 \mathrm{mmHg}$; inability to provide written informed consent; and any other condition which at the Investigator's discretion, was believed may present a safety risk or impact on the feasibility of the study results. Written informed consent was obtained before any study-specific procedures. The study was undertaken on the ward during the hospital admission. Ethics approval was obtained from the Health and Disability Ethics Committee, New Zealand (Reference 14/NTB/200). The full study protocol (original and updated version) can be found on the OLS (see Additional file 1 and 2).

\section{Intervention}

After written consent, participants had continuous $\mathrm{PtCO}_{2}$ and heart rate monitoring using the SenTec (SenTec AG, Switzerland) device and oxygen saturation $\left(\mathrm{SpO}_{2}\right)$ measured by pulse oximetry (Novametrix 512, Respironics, Carlsbad, USA). Participants were randomised to receive two nebulisations, both driven either by air or oxygen, at $8 \mathrm{~L} / \mathrm{min}$, each delivered over $15 \mathrm{~min}$ with a five minute break in-between. Randomisation was 1:1 by a block randomised computer generated sequence (block size six), provided in sealed opaque envelopes by the study statistician who was independent of recruitment and assessment of participants.

The participants and blinded investigator, who recorded heart rate and $\mathrm{PtCO}_{2}$ were masked to the randomised treatments. If both oxygen and air ports were available in hospital on the wall behind the participant, these were used for driving nebulisation. If only oxygen ports were available, identical portable oxygen and air cylinders were placed behind the participant's bed prior to randomisation and used instead. Both the participant and blinded investigator faced forward for the full duration of the study. In addition, the blinded investigator sat towards the end of the bed - ahead of the participant, such that they could not see the participant's interventions. Likewise, the blinded investigator and patient could not view the $\mathrm{SpO}_{2}$ on the Sentec device, as this was covered during the interventions, or the pulse oximeter which could only be viewed by the unblinded investigator. Interaction between blinded and unblinded investigators would only occur if a rise in $\mathrm{PtCO}_{2}$ of $\geq 10 \mathrm{mmHg}$ was demonstrated (a predefined safety criterion to abort intervention).

An initial 15 min wash-in and titration period was administered by the unblinded investigator using nasal cannulae, if required, to ensure that participant's $\mathrm{SpO}_{2}$ were within 88 to $92 \%$. If saturations were $\geq 88 \%$ on room air, no supplemental oxygen was required. Randomisation was performed after the 15 min wash-in period, when both patient and blinded investigator were already in a forward-facing position to maintain blinding. The unblinded investigator recorded $\mathrm{SpO}_{2}$ on a separate pulse-oximeter from then onwards.

Immediately before the first nebulisation, denoted by the baseline reading at time-point zero, $\mathrm{PtCO}_{2}, \mathrm{SpO}_{2}$ and heart rate were recorded. Participants then received two administrations of $2.5 \mathrm{mg}$ salbutamol by nebulisation, 
delivered by either air or oxygen - each for 15 min duration at a flow rate of $8 \mathrm{~L} / \mathrm{min}$. Hudson RCI Micro Mist Nebuliser Masks (Hudson RCI, Durham, North Carolina, USA) were used. The nebulisations were delivered by the unblinded investigator at time zero and at $20 \mathrm{~min}$, allowing for a five minute interval between nebulisations. Recordings were continued for $45 \mathrm{~min}$ after completion of the last nebulisation (80 min after baseline). Measurements of $\mathrm{PtCO}_{2}, \mathrm{SpO}_{2}$ and heart rate were recorded at five minute intervals, and at six minutes after the start of each nebulisation, in view of the British Thoracic Society (BTS) guideline's recommendation for limiting oxygen-driven nebulisation to six-minutes in ambulance care, if air-driven nebulisation is unavailable [6].

Immediately before the first nebulisation and just before completion of the second nebulisation, at $35 \mathrm{~min}$, a capillary blood gas sample was taken from the fingertip for measurement of $\mathrm{PcapCO}_{2}$ and $\mathrm{pH}$.

\section{Oxygen delivery}

During the wash-in and between the nebulisations oxygen was titrated, if required, via nasal prongs to maintain oxygen saturations between 88 to $92 \%$. Participants in the air-driven group who were receiving oxygen at the start of nebulisation continued to receive titrated supplemental oxygen via nasal prongs underneath the nebuliser mask. Those in the oxygen-driven group had the prongs removed at the start, and reapplied after the completion of each nebulisation. At $35 \mathrm{~min}$, oxygen was delivered via nasal prongs to participants at the flow rate they last received during titration (i.e. at $35 \mathrm{~min}$ and $20 \mathrm{~min}$ in the air-driven and oxygen-driven groups, respectively). From 35 min until $80 \mathrm{~min}$, the oxygen flow rate was only increased (or initiated) if a participant's $\mathrm{SpO}_{2}$ fell below $85 \%$.

\section{Outcomes}

The primary outcome was originally planned to be $\mathrm{PcapCO}_{2}$ at $35 \mathrm{~min}$, at completion of the second nebulisation. However, after the first 14 participants had been studied, it was evident that obtaining adequate amounts of blood to fill the capillary tubes from some participants was difficult. At this stage of recruitment 4/14 (29\%) of participants had missing data. The primary outcome variable was therefore changed to $\mathrm{PtCO}_{2}$ at $35 \mathrm{~min}$, with $\mathrm{PcapCO}_{2}$ at 35 min reverting to a secondary outcome variable. Other secondary outcomes were the individual $\mathrm{PtCO}_{2}$ measurements at each time point; the proportion of participants who had a rise in $\mathrm{PtCO}_{2}$ or $\mathrm{PcapCO}_{2}$ of $\geq 4$ and $\geq 8 \mathrm{mmHg}$; capillary $\mathrm{pH}$ at $35 \mathrm{~min}$, and heart rate and $\mathrm{SpO}_{2}$ measurements at each time point.

\section{Sample size calculation and statistical analysis}

A rise in $\mathrm{PtCO}_{2}$ from baseline of $\geq 4 \mathrm{mmHg}$ is considered a physiologically significant change and $\geq 8 \mathrm{mmHg}$ a clinically significant change, based on previous criteria $[7,8]$. In our study of oxygen versus air-driven nebulisers in stable COPD patients, the standard deviation (SD) of baseline $\mathrm{PtCO}_{2}$ was $5.5 \mathrm{mmHg}$ [3]. With $90 \%$ power and alpha of $5 \%, 82$ patients were required to detect a $4 \mathrm{mmHg}$ difference. Assuming a drop-out rate of $10 \%$ our target recruitment was 90 patients.

The primary analysis used a mixed linear model with fixed effects of the baseline $\mathrm{PtCO}_{2}$, time, the randomised intervention, and a time by intervention interaction term; to estimate the difference between randomised treatments at $35 \mathrm{~min}$. A power exponential in time correlation structure was used for the repeated measurements. The secondary outcome variables of $\mathrm{PtCO}_{2}$ at the other time points, $\mathrm{SpO}_{2}$ and heart rate used similar mixed linear models. $\mathrm{PcapCO}_{2}$ and $\mathrm{pH}$ were compared by Analysis of Covariance with the baseline measurement as a continuous co-variate. As a post-hoc analysis we compared the difference in $\mathrm{PtCO}_{2}$ between the 15 and $6 \mathrm{~min}$, and the 35 and $26 \mathrm{~min}$ time points.

Comparison of categorical variables, $\mathrm{PtCO}_{2}$ or $\mathrm{PcapCO}_{2}$ change of $\geq 4$ and $8 \mathrm{mmHg}$, was by estimation of a risk difference, and Fishers' exact test. As a post-hoc analysis we also compared the difference in paired proportions for those with $\mathrm{PtCO}_{2}$ change of $\geq 4 \mathrm{mmHg}$ in the oxygen arm only using McNemar's test and an appropriate estimate for the difference in paired proportions. The time for $\mathrm{PtCO}_{2}$ to return to baseline during the observation period (defined as the time until the $\mathrm{PtCO}_{2}$ was first equal to or below the baseline value, between 40 and $80 \mathrm{~min}$ ), was compared using Kaplan-Meier survival curves and a Cox Proportional Hazards model. A simple t-test was used to compare the lowest value of the $\mathrm{SpO}_{2}$ between 40 and $80 \mathrm{~min}$, compared to baseline. SAS version 9.4 was used.

\section{Results}

Patients

The trial recruited between May 14th 2015 and June 29th 2016. The CONSORT diagram of the flow of the 90 recruited participants through the trial is shown in Fig. 1. One participant withdrew after $18 \mathrm{~min}$ of air-driven nebulisation because of feeling flushed, and so complete data was available for $\mathrm{PtCO}_{2}$ for 89 participants. The baseline $\mathrm{PtCO}_{2}$ for this participant was $34.3 \mathrm{mmHg}$ and at the time of withdrawal it was $34.6 \mathrm{mmHg}$. Oxygen-driven nebulisation was stopped in another participant at $27 \mathrm{~min}$ when the $\mathrm{PtCO}_{2}$ rose by $>10 \mathrm{mmHg}$ from baseline, a pre-defined safety criterion. The baseline $\mathrm{PtCO}_{2}$ for this participant was $43.4 \mathrm{mmHg}$ and at the time of withdrawal it was $54.1 \mathrm{mmHg}$. This participant had study measurements continued after this for the full duration of the study. No clinical adverse events were noted during the intervention periods. 


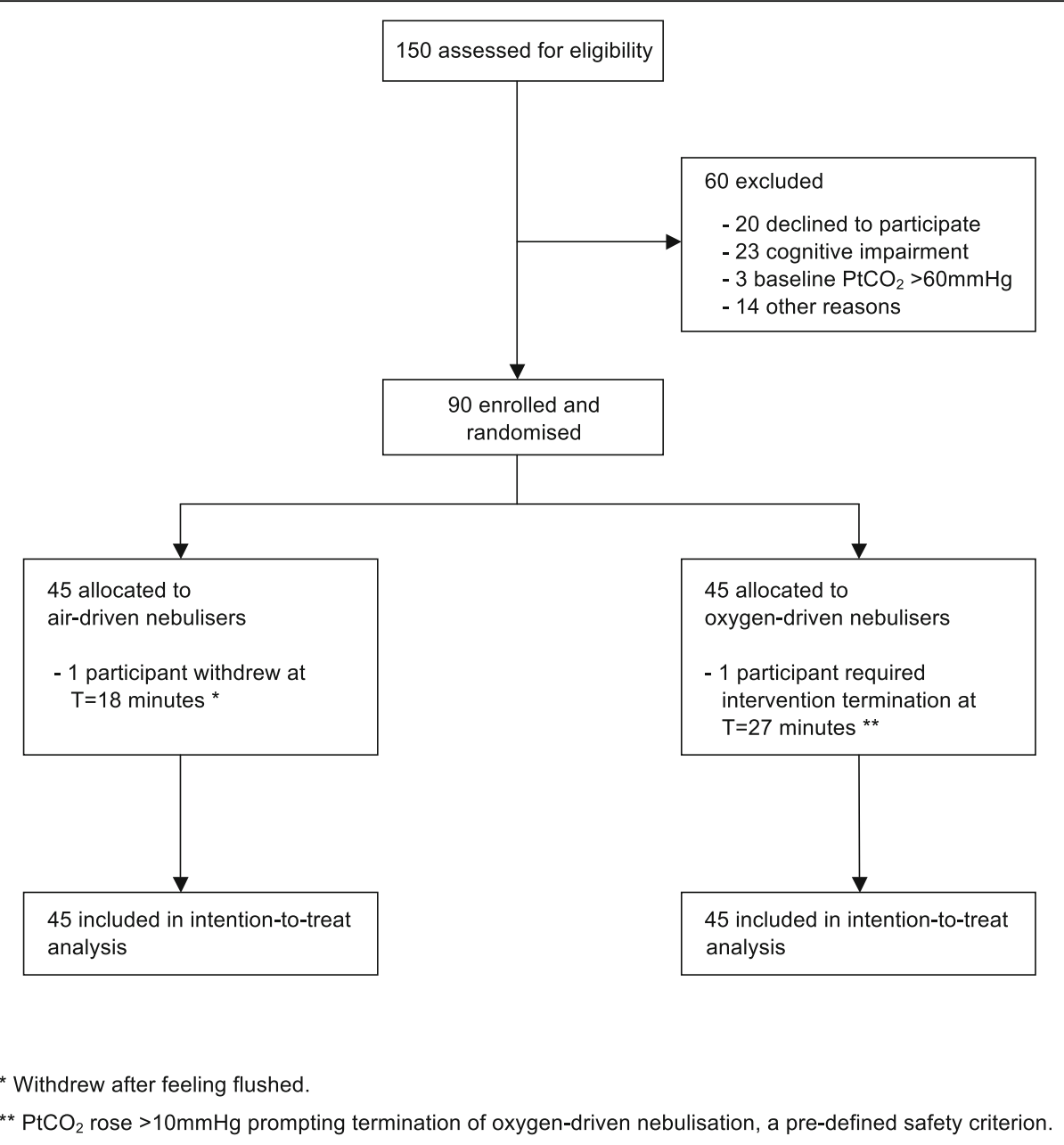

Fig. 1 Participant flow through the study and allocation of interventions

A summary of baseline participant characteristics are shown in Table 1 . Participants predominantly had severe airflow obstruction with a mean $\mathrm{FEV}_{1}$ of $34.5 \%$ predicted. The mean (range) baseline $\mathrm{PtCO}_{2}$ was $37.6 \mathrm{mmHg}(24.3$ to $58.5 \mathrm{mmHg}$ ), and mean $\mathrm{SpO}_{2}$ was $93 \%$. Patients randomised to the oxygen group were more likely to have required assisted ventilation previously. The mean (SD) time for the nebulised salbutamol to dissipate from the chamber was 5.2 (1.2) minutes.

\section{$\mathrm{PtCO}_{2}$}

The mean (SD) change in $\mathrm{PtCO}_{2}$ after 35 min was 3.4 (1.9) $\mathrm{mmHg}$ in the oxygen group $(n=45)$, compared to 0.1 (1.4) $\mathrm{mmHg}$ in the air group $(n=44)$. The difference (95\% CI) in $\mathrm{PtCO}_{2}$ for oxygen compared to air-driven nebulisations after $35 \mathrm{~min}$ was $3.3 \mathrm{mmHg}$ (2.7 to 3.9 ), $p<0.001$. (Table 2 and Fig. 2). After adjustment for baseline $\mathrm{PtCO}_{2}$, a history of assisted ventilation, previous hypercapnia and baseline $\mathrm{SpO}_{2}$, were not associated with the $\mathrm{PtCO}_{2}$ at $35 \mathrm{~min}$ in either randomised group.
In $18 / 45(40 \%)$ participants receiving oxygen-driven nebulisation, $\mathrm{PtCO}_{2}$ increased from baseline by $\geq 4 \mathrm{mmHg}$ at some stage during the intervention compared to none of the participants receiving air-driven nebulisation, risk difference (95\% CI) 40\% (25.7 to 54.3), $\mathrm{p}<0.001$. The full data description and comparisons at each time point are shown in the OLS. Two participants receiving oxygen-driven nebulisation had a rise in $\mathrm{PtCO}_{2} \geq 8 \mathrm{mmHg}$, one of whom required intervention termination, exceeding the predefined safety criterion of a rise $\geq 10 \mathrm{mmHg}$ from baseline.

The estimate $(95 \% \mathrm{CI})$ of the time-related difference, 15 min minus six minutes, for oxygen compared to air, was $0.73 \mathrm{mmHg}$ ( 0.11 to 1.35 ), $P=0.021$; and for $35 \mathrm{~min}$ minus $26 \mathrm{~min}, 0.43 \mathrm{mmHg}$ ( -0.19 to 1.06 ), $P=0.17$. In the oxygen treatment arm the proportion of patients in whom the $\mathrm{PtCO}_{2}$ increased from baseline by $\geq 4 \mathrm{mmHg}$ at $6 \mathrm{~min}$ was less than the proportion at $15 \mathrm{~min}$ : 6/45 $(13.3 \%)$ and $13 / 45(28.9 \%)$ respectively, paired difference in proportions $(95 \% \mathrm{CI}) 15.6 \%$ (3.3 to 27.8 ), $P=0.013$ 
Table 1 Participant Characteristics

\begin{tabular}{|c|c|c|c|}
\hline & \multicolumn{2}{|c|}{ Mean (SD) } & $P$ \\
\hline & Oxygen $N=45^{a}$ & Air $N=45^{a}$ & \\
\hline Age (years) & $70 \cdot 4(10 \cdot 3)$ & $72 \cdot 3(8.3)$ & 0.34 \\
\hline $\begin{array}{l}\text { Age at diagnosis } \\
\text { of COPD (years) }\end{array}$ & $58 \cdot 6(12 \cdot 1) N=40$ & $58.8(12.2) N=44$ & 0.92 \\
\hline BMI $\left(\mathrm{kg} / \mathrm{m}^{2}\right)$ & $27 \cdot 2(7 \cdot 7)$ & $25.5(8.9)$ & 0.33 \\
\hline Smoking pack years & $39 \cdot 3(31 \cdot 1)$ & $51 \cdot 2(39 \cdot 2)$ & 0.11 \\
\hline $\mathrm{FEV}_{1}(\mathrm{~L})$ & $0.81(0.33) N=35$ & $0.85(0.31) N=37$ & 0.69 \\
\hline $\mathrm{FEV}_{1} \%$ predicted & $35.0(11.5) N=35$ & $34.0(11 \cdot 8) N=37$ & 0.73 \\
\hline mMRC & $2.38(1.09)$ & $2.33(1.04)$ & 0.84 \\
\hline
\end{tabular}

Baseline Transcutaneous Data

$\begin{array}{llll}\mathrm{PtCO}_{2}(\mathrm{mmHg}) & 38.0(7.7) & 37.2(6.8) & 0.59 \\ \mathrm{SpO}_{2}(\%) & 92.6(2.4) & 92.6(2.3) & 0.93 \\ \text { Heart Rate (per minute) } & 89.6(15.7) & 87.0(16.0) & 0.89\end{array}$

Baseline capillary blood gas

$\begin{array}{llll}\mathrm{pH} & 7.42(0.04) \mathrm{N}=43 & 7.44(0.03) N=41 & 0.11 \\ \mathrm{PcapCO}_{2}(\mathrm{mmHg}) & 40.2(7.0) \mathrm{N}=43 & 38.5(5.9) N=41 & 0.23 \\ & \mathrm{~N} / 45(\%) & P \\ & \text { Oxygen } & \text { Air } & \end{array}$

\begin{tabular}{|c|c|c|}
\hline Male & $17(38)$ & $24(53)$ \\
\hline
\end{tabular}

\begin{tabular}{llll} 
European & $24(53)$ & $31(69)$ & \\
Māori & $7(16)$ & $4(9)$ & \\
Pacific & $5(11)$ & $4(9)$ & \\
Other & $9(20)$ & $6(13)$ & 0.02 \\
Previous Ventilation (ever) & $12(27)$ & $3(7)$ & 0.03 \\
Previous Ventilation Type & & & \\
NIV & $10(22)$ & $3(7)$ & \\
$\quad$ Intubation & $2(4)$ & $0(0)$ & 0.29 \\
Previous hypercapnia & $23(51)$ & $17(38)$ & 0.99 \\
Home Oxygen & $2(4)$ & $1(2)$ & 0.10 \\
Home Nebulisers & $5(11)$ & $12(27)$ & \\
Comorbidities & & & 0.20 \\
Heart Failure & $8(18)$ & $3(7)$ & 0.27 \\
Asthma & $6(13)$ & $2(4)$ & 0.99 \\
Bronchiectasis & $3(7)$ & $4(9)$ & \\
\hline
\end{tabular}

COPD Chronic Obstructive Pulmonary Disease, BMI Body Mass Index, FEV Forced Expiratory Volume in $1 \mathrm{~s}$ at time of randomisation, $m M R C$ Modified Medical Research Council dyspnea scale, $P t \mathrm{CO}_{2}$ Transcutaneous partial pressure of carbon dioxide, $\mathrm{SpO}_{2}$ peripheral oxygen saturation, $\mathrm{PcapCO}_{2}$ Capillary partial pressure of carbon dioxide, NIV non-invasive ventilation aUnless indicated

(Additional file 1: Table S1). The proportion of patients in whom the $\mathrm{PtCO}_{2}$ increased from baseline by $\geq 4 \mathrm{mmHg}$ at $26 \mathrm{~min}(6 \mathrm{~min}$ into the second oxygen-driven nebulisation) was also less than the proportion at 35 min (completion of the second oxygen-driven nebulisation), although this difference was not statistically significant: 10/45 (22\%) and 14/45 (31\%) respectively, paired difference in proportions (95\% CI) $8.9 \%$ ( -3.3 to 20.9 ), $P=0.15$.

The median (25th to 75 th percentile) time taken for $\mathrm{PtCO}_{2}$ to return to baseline after cessation of the second nebulisation was 40 (40 to 45) minutes in the air group compared to 50 (45 to 50) minutes in the oxygen group, hazard ratio (95\% CI) 1.59 (1.01 to 2.52$), P=0.047$.

\section{$\mathrm{PcapCO}_{2}$ and $\mathrm{pH}$}

Data summaries for capillary blood gas sampling are shown in Table 3 . The difference $(95 \%$ CI) between oxygen and air for $\mathrm{PcapCO}_{2}$ after $35 \mathrm{~min}$ was $2.0 \mathrm{mmHg}$ (1.1 to 2.8$), p<0.001$. Thirteen $(31.7 \%)$ participants receiving oxygen had a rise in $\mathrm{PcapCO}_{2}$ of $\geq 4 \mathrm{mmHg}$ compared with three $(7.7 \%)$ receiving air; risk difference (95\% CI) $24 \%$ (7.5 to 40.5 ), $p=0.01$. In addition to the two participants in whom the $\mathrm{PtCO}_{2}$ increased by $\geq 8 \mathrm{mmHg}$, there were two additional participants with capillary data receiving oxygen who had a rise in $\mathrm{PcapCO}_{2}$ of $\geq 8 \mathrm{mmHg}$ and none from the air group. The mean $(95 \% \mathrm{CI})$ difference in $\mathrm{pH}$ after 35 min was 0.015 units $(0.008$ to 0.024 , p $<0.001)$ lower for oxygen nebulisation compared to air. One participant experienced a reduction in $\mathrm{pH}$ of 0.06 units (from 7.38 to 7.32 ) in association with a rise in $\mathrm{PcapCO}_{2}$ of $9 \mathrm{mmHg}$ ( 55 to $64 \mathrm{mmHg}$ ).

\section{$\mathrm{SpO}_{2}$ and heart rate}

The $\mathrm{SpO}_{2}$ was higher throughout both the nebulisation and initial washout periods in the oxygen compared with the air group (see Additional file 3: Table S2). Figure 3 shows the trend for the $\mathrm{SpO}_{2}$ in the oxygen group to fall below that of the air group after cessation of the second nebulisation. At the end of the observation period (80 $\mathrm{min}$ ), the $\mathrm{SpO}_{2}$ was lower in the oxygen group (difference $-1.22 \%$, 95\% CI -2.04 to $-0.39, p=0.004$ ). The maximum reduction in $\mathrm{SpO}_{2}$ from baseline was $0.8 \%$ (95\% CI -0.2 to $1.7, P=0.10$ ) lower after oxygen compared with air nebulisation. The heart rate was slower in the oxygen group at $35 \mathrm{~min}$ by $3.3 \mathrm{bpm}$ (95\% CI 0.31 to 6.25), $p=0.031$ (see Additional file 1: Table S3).

\section{Methods of $\mathrm{PCO}_{2}$ measurement}

Due to the requirement to change the primary outcome measure, a post-hoc analysis was undertaken to compare the two methods of measuring $\mathrm{PaCO}_{2}$. Based on data for 80 paired $\mathrm{PtCO}_{2}$ and $\mathrm{PcapCO}_{2}$ measurements at baseline and $35 \mathrm{~min}$, the mean (SD) change in $\mathrm{PtCO}_{2}$ was $1.7 \mathrm{mmHg}$ (2.2) with a range of -2.5 to $8.0 \mathrm{mmHg}$, and the mean (SD) change in $\mathrm{PcapCO}_{2}$ was $1.7 \mathrm{mmHg}(2.3)$, with a range -3.0 to $9.0 \mathrm{mmHg}$. The estimate of bias for change in $\mathrm{PcapCO}_{2}$ minus $\mathrm{PtCO}_{2}$ was $-0.03 \mathrm{mmHg}$ (95\% CI -0.44 to 0.38 ), $P=0.89$. The limits of agreement between $\mathrm{PtCO}_{2}$ and $\mathrm{PcapCO}_{2}$ were $+/-3.8 \mathrm{mmHg}$ for each individual measurement obtained. 
Table $2 \mathrm{PtCO}_{2}$ by time and randomised group

\begin{tabular}{|c|c|c|c|c|c|}
\hline \multirow[t]{3}{*}{ Action } & \multirow[t]{3}{*}{ Time } & \multicolumn{2}{|c|}{$\mathrm{PtCO}_{2}$ Mean (SD) } & \multirow{3}{*}{$\begin{array}{l}\text { Oxygen minus air } \\
(95 \% \mathrm{Cl})\end{array}$} & \multirow[t]{3}{*}{$P$} \\
\hline & & \multicolumn{2}{|c|}{ [N $=45$ for each unless specified $]$} & & \\
\hline & & Oxygen & Air & & \\
\hline Baseline & 0 & $38.0(7 \cdot 7)$ & $37 \cdot 2(6 \cdot 8)$ & & \\
\hline \multirow[t]{5}{*}{ 1st nebulisation } & 5 & $39.9(8.3)$ & $37.0(7 \cdot 1)$ & $2.10(1.49$ to 2.71$)$ & $<0.001$ \\
\hline & 6 & $40 \cdot 1(8 \cdot 4)$ & $37.0(7.1)$ & 2.24 (1.63 to 2.86$)$ & $<0.001$ \\
\hline & 10 & $40 \cdot 8(8 \cdot 6)$ & $37 \cdot 2(6 \cdot 9)$ & $2.76(2.15$ to 3.37$)$ & $<0.001$ \\
\hline & 15 & $41 \cdot 1(8 \cdot 8)$ & $37.3(6.9)$ & 2.97 (2.36 to 3.59$)$ & $<0.001$ \\
\hline & 20 & $38.6(7.8)$ & $37.0(6.4)^{\mathrm{a}}$ & $0.86(0.25$ to 1.48$)$ & 0.006 \\
\hline \multirow[t]{4}{*}{ 2nd nebulisation } & 25 & $40 \cdot 5(8 \cdot 3)$ & $36 \cdot 8(6 \cdot 8)^{\mathrm{b}}$ & $2.77(2.15$ to 3.39$)$ & $<0.001$ \\
\hline & 26 & $40.6(8.4)$ & $36.8(6.8)^{\mathrm{b}}$ & 2.88 (2.26 to 3.50$)$ & $<0.001$ \\
\hline & 30 & $41 \cdot 1(8.5)$ & $37.1(6.6)^{\mathrm{a}}$ & 3.20 (2.59 to 3.82$)$ & $<0.001$ \\
\hline & 35 & $41 \cdot 3(8 \cdot 6)$ & $37.3(6.5)^{\mathrm{a}}$ & 3.31 (2.70 to 3.93 ) & $<0.001$ \\
\hline \multirow[t]{9}{*}{ Observation period } & 40 & $39.0(8.1)^{\mathrm{a}}$ & $37.0(6.7)^{\mathrm{a}}$ & $1.14(0.52$ to 1.76$)$ & $<0.001$ \\
\hline & 45 & $38.1(7.5)$ & $36 \cdot 7(6 \cdot 3)^{\mathrm{a}}$ & $0.61(-0.01$ to 1.22$)$ & 0.053 \\
\hline & 50 & $37.9(7.4)$ & $36 \cdot 6(6 \cdot 2)^{a}$ & $0.59(-0.02$ to 1.21$)$ & 0.059 \\
\hline & 55 & $37.9(7.3)$ & $36 \cdot 6(6 \cdot 0)^{\mathrm{a}}$ & $0.51(-0.10$ to 1.13$)$ & $0 \cdot 1$ \\
\hline & 60 & $37.9(7.3)$ & $36.7(6 \cdot 1)^{\mathrm{a}}$ & $0.51(-0.10$ to 1.13$)$ & $0 \cdot 1$ \\
\hline & 65 & $38 \cdot 1(7 \cdot 2)$ & $36.7(6 \cdot 1)^{\mathrm{a}}$ & $0.63(0.01$ to 1.25$)$ & 0.045 \\
\hline & 70 & $37.5(6.5)^{\mathrm{a}}$ & $36.7(6 \cdot 1)^{a}$ & $0.60(-0.02$ to 1.21$)$ & 0.059 \\
\hline & 75 & $37.8(6 \cdot 8)$ & $36 \cdot 7(6 \cdot 1)^{a}$ & $0.42(-0.20$ to 1.03$)$ & 0.18 \\
\hline & 80 & $37.9(6.9)$ & $36 \cdot 7(6 \cdot 3)^{a}$ & $0.40(-0.21$ to 1.02$)$ & 0.2 \\
\hline
\end{tabular}

Air Air-driven nebuliser group, Oxygen Oxygen-driven nebuliser group, $\mathrm{PtCO}_{2}$ Transcutaneous partial pressure of carbon dioxide

${ }^{\mathrm{a}} \mathrm{N}=44$

${ }^{\mathrm{b}} \mathrm{N}=43$

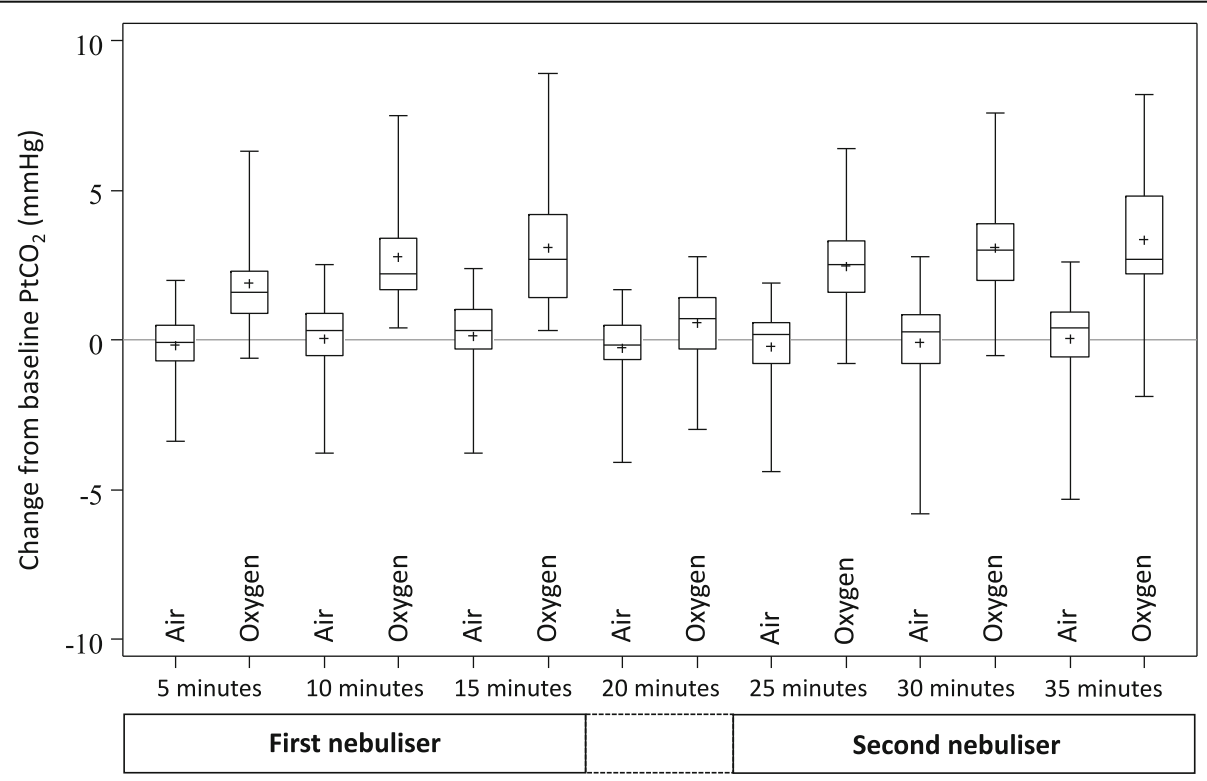

Air: Air-driven nebuliser group; Oxygen: Oxygen-driven nebuliser group;

$\mathrm{PtCO}_{2}$ : Transcutaneous carbon dioxide tension

Fig. $2 \mathrm{PtCO}_{2}$ change from baseline $(T=0)$ to $T=35$ min. Mean $\mathrm{PtCO}_{2}$ with error bars showing one $\mathrm{SD}$, by time and intervention 
Table 3 Capillary blood gas measurements according to randomised treatment

\begin{tabular}{|c|c|c|c|c|}
\hline \multirow[t]{2}{*}{ Time (mins) } & \multicolumn{2}{|c|}{$\mathrm{P}_{\text {cap }} \mathrm{CO}_{2}$ Mean (SD) } & \multirow[t]{2}{*}{ Difference $^{\mathrm{a}}(95 \% \mathrm{Cl})$} & \multirow[t]{2}{*}{$P$} \\
\hline & Oxygen & Air & & \\
\hline 0 & $40 \cdot 2(7 \cdot 0) N=43$ & $38.5(5.9) N=41$ & - & - \\
\hline 35 & $42 \cdot 6(8 \cdot 3) N=41$ & $39.0(6.4) N=39$ & $2 \cdot 0(1.1$ to $2 \cdot 8)$ & $<0.001$ \\
\hline \multirow[t]{2}{*}{ Time (mins) } & \multicolumn{2}{|c|}{ pH Mean (SD) } & Difference $^{\mathrm{b}}(95 \% \mathrm{Cl})$ & $P$ \\
\hline & Oxygen & Air & & \\
\hline 0 & $7.42(0.04) N=43$ & $7.44(0.03) N=41$ & - & - \\
\hline 35 & $7.41(0.04) N=41$ & $7.43(0.04) N=39$ & $-0.015(-0.024$ to -0.008$)$ & $<0.001$ \\
\hline
\end{tabular}

$P_{\text {cap }} \mathrm{CO}_{2}$ Capillary partial pressure of carbon dioxide

${ }^{\mathrm{a}} \mathrm{P}_{\text {cap }} \mathrm{CO}_{2}$ at $35 \mathrm{~min}$, adjusted for baseline

${ }^{\mathrm{b}} \mathrm{pH}$ at $35 \mathrm{~min}$, adjusted for baseline

\section{Discussion}

In this study, oxygen-driven nebulisation increased the $\mathrm{PtCO}_{2}$ in hospital in-patients with an AECOPD compared with air-driven nebulisation. Despite the small mean increase in $\mathrm{PtCO}_{2}$ of $3.4 \mathrm{mmHg}$, the physiological relevance of this response is suggested by the increase in $\mathrm{PtCO}_{2}$ of at least $4 \mathrm{mmHg}$ in 18/45 (40\%) of participants receiving oxygen-driven nebulisation, whereas no patient had an increase of $4 \mathrm{mmHg}$ or more following air-driven nebulisation. The clinical relevance of this physiological response is suggested by the requirement to withdraw one participant during the second oxygen-driven nebulisation due to the $\mathrm{PtCO}_{2}$ increasing by $>10 \mathrm{mmHg}$, and the increase of $\mathrm{PtCO}_{2}$ or $\mathrm{PcapCO}_{2}$ of at least $8 \mathrm{mmHg}$ in $4 / 45$ (9\%) patients receiving oxygen-driven nebulisation, one of whom had a fall in $\mathrm{pH}$ of 0.06 into the acidotic range (7.32). These findings suggest that air-driven nebulised bronchodilator therapy represents an important component of the conservative titrated oxygen regimen which has been shown to reduce the risk of hypercapnia, acidosis and mortality in AECOPD [1].

There are a number of methodological issues relevant to the interpretation of the study findings. Both the randomised controlled design and double-blinding of this study allow for robust and reliable data capture. The length of the nebuliser regimen was chosen to ensure adequate time for complete nebulisation to occur, and to replicate 'real-world' back to back treatments in the acute setting, by using two nebulisations separated by five minutes. It is possible that the magnitude of the

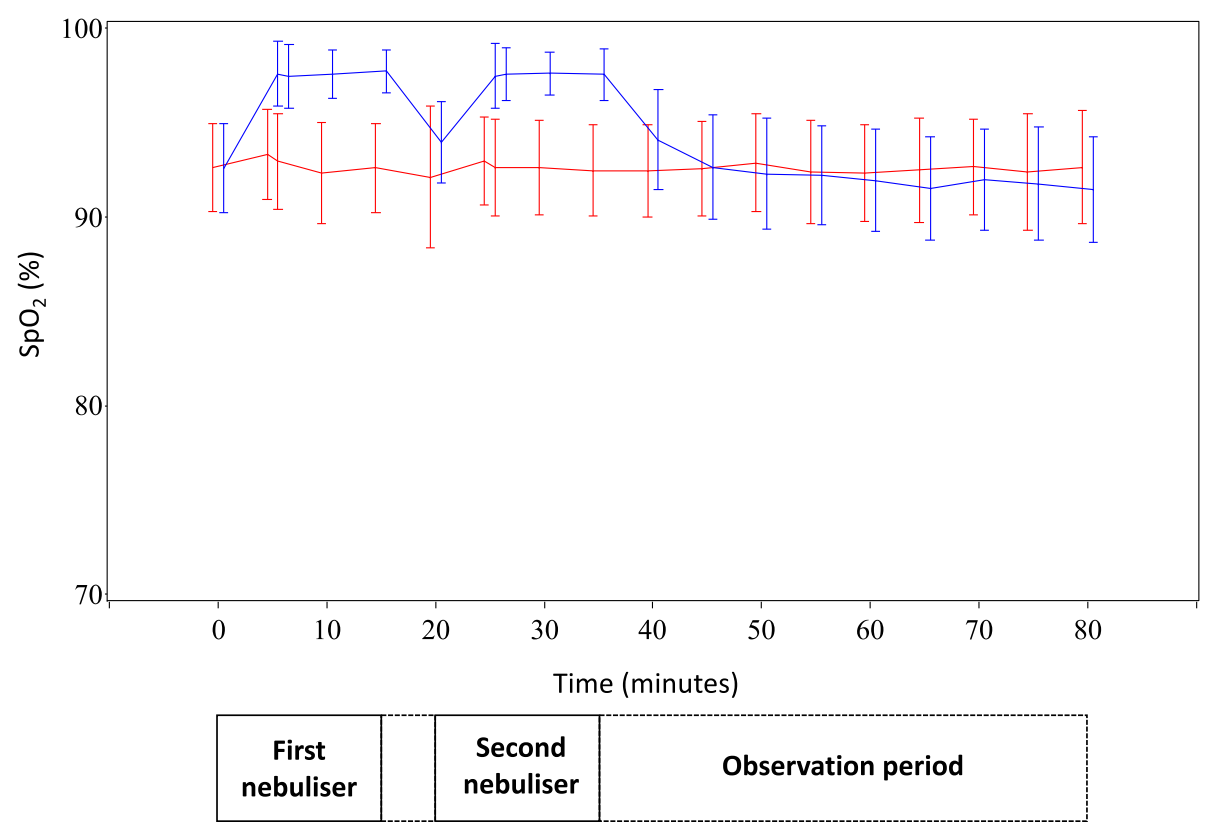

$\mathrm{SpO}_{2}$ : Oxygen saturation measured by pulse oximeter

Fig. 3 Time-course of $\mathrm{SpO}_{2}$ throughout study period (Blue = Oxygen-driven nebuliser group, Red = Air-driven nebuliser group) 
differences in $\mathrm{PCO}_{2}$ and $\mathrm{pH}$ may be even larger with continuous nebulisation which may occur in patients with severe exacerbations not responding to initial treatment or if the nebuliser is inadvertently left in place. The safety-based exclusion criteria of a baseline $\mathrm{PtCO}_{2}>$ $60 \mathrm{mmHg}$ and an oxygen requirement of $\geq 4 \mathrm{~L} /$ minute (to maintain target $\mathrm{SpO}_{2}$ of 88 to $92 \%$ ), effectively excluded patients with the most severe exacerbations of COPD.

Whilst respiratory rate and neurological symptoms were not formally assessed as outcome measures, no adverse events were identified during the interventions. However, we acknowledge that if changes in $\mathrm{PCO}_{2}$ and $\mathrm{pH}$ of this magnitude occurred in more severe patients at the time of their presentation, they would have been at risk of symptoms of hypercapnia and respiratory acidosis, and the requirement to escalate treatment.

The original primary outcome measure and time of measurement was $\mathrm{PcapCO}_{2}$ after $35 \mathrm{~min}$. Following the first 14 study participants, it was evident that obtaining adequate amounts of blood to fill the capillary tubes from some participants was difficult or impossible to the extent that 4 out of 14 participants had one or more missed samples. For this reason, the primary outcome was changed to $\mathrm{PtCO}_{2}$ after $35 \mathrm{~min}$. In other words, the method of capturing the change in $\mathrm{PCO}_{2}$ was revised, rather than the outcome itself. $\mathrm{PtCO}_{2}$ monitoring enabled continuous assessment to be undertaken, and is accurate in AECOPD, [9] and other acute settings [10-12]. The validity of this method was confirmed by the post hoc analysis of 80-paired samples, where each capillary blood gas sample obtained had a corresponding $\mathrm{PtCO}_{2}$ measurement at the same time-point. This showed that the difference between the $\mathrm{PcapCO}_{2}$ and $\mathrm{PtCO}_{2}$ in the mean change from baseline was $-0.03 \mathrm{mmHg}$ with $95 \%$ confidence intervals of -0.44 to $0.38 \mathrm{mmHg}$. This data suggests that the use of $\mathrm{PtCO}_{2}$ measurements did not adversely affect our ability to determine change in $\mathrm{PcapCO}_{2}$ from baseline.

We did not investigate the potential mechanisms by which oxygen driven nebulisation increases $\mathrm{PtCO}_{2}$. However as demonstrated in mechanistic studies of oxygen therapy in COPD, it is likely to be due to the combination of a reduction in respiratory drive, release of hypoxic pulmonary vasoconstriction, absorption atelectasis, and the Haldane effect $[13,14]$. Furthermore, the study was not designed to assess costs related to each regimen, however it is reasonable to assume that improved clinical outcomes seen by avoiding a rise in $\mathrm{PtCO}_{2}$ and associated acidosis, would lead to a reduction in healthcare costs.

The findings from our study complement those of our previous randomised controlled trial of a similar design in stable COPD patients in the clinic setting, in which there was a mean $\mathrm{PtCO}_{2}$ difference between the oxygen- and air-driven nebulisation treatment arms of $3.1 \mathrm{mmHg}$ (95\% CI 1.6 to 4.5 ), $p<0.001$, after $35 \mathrm{~min}$. [3] In that study one of the 24 subjects was withdrawn due to an increase in $\mathrm{PtCO}_{2}$ of $10 \mathrm{mmHg}$ after $15 \mathrm{~min}$ of the first oxygen-driven nebulisation. As with the previous study, an increase in $\mathrm{PtCO}_{2}$ occurred within $5 \mathrm{~min}$, indicating the rapid time course of this physiological response. We had anticipated a greater effect in this current study as the patients had acute rather than stable COPD however the magnitude of the effect was similar, probably reflecting the similar severity of airflow obstruction, with a mean predicted $\mathrm{FEV}_{1}$ of $35 \%$ and $27 \%$ in this and the previous study respectively.

The two previous open crossover studies of inpatients with AECOPD both showed oxygen-driven nebulisation worsened hypercapnia in patients with Type 2 respiratory failure [4, 5]. Gunawardena et al. [4] studied 16 patients with COPD and reported that only those with carbon dioxide retention at baseline $(n=9)$ demonstrated a rise in $\mathrm{PaCO}_{2}$ after $15 \mathrm{~min}$ (mean of $7.7 \mathrm{mmHg}$ ), and one patient had a rise of $22 \mathrm{mmHg}$. Similarly, O'Donnell et al [5] reported that $6 / 10$ patients, all with carbon dioxide retention at baseline, showed a rise in $\mathrm{PaCO}_{2}$ after $10 \mathrm{~min}$ (mean of $12.5 \mathrm{mmHg}$ ).

The current BTS guidelines recommend air-driven nebulisation and, if this is not available in the ambulance service, the maximum use of $6 \mathrm{~min}$ for an oxygen-driven nebuliser. This is based on the rationale that most of the nebulised medication will have been delivered, and is categorised as grade D evidence [6]. We observed the mean time for dissipation of salbutamol solution from the nebuliser chamber of $5.2 \mathrm{~min}$ confirming that $6 \mathrm{~min}$ is adequate for salbutamol delivery. The proportion of participants with a $\mathrm{PtCO}_{2}$ increase $\geq 4 \mathrm{mmHg}$ was lower after 6 min than 15 min, suggesting some amelioration of risk with the shorter nebulisation treatment. Alternative methods of bronchodilator delivery include air-driven nebulisers or multiple metered dose inhaler actuations via a spacer [15].

The potential for rebound hypoxia after abrupt cessation of oxygen therapy has been observed both in the treatment of asthma and COPD [9, 16, 17]. We identified some evidence consistent with this phenomenon which is a potentially important yet poorly recognised clinical issue.

\section{Conclusions}

In summary, air-driven nebulisation avoids the potential risk of increasing the $\mathrm{PaCO}_{2}$ associated with oxygendriven bronchodilator administration in AECOPD. We propose that air-driven bronchodilator nebulisation is preferable to oxygen-driven nebulisation in AECOPD, and that when the use of oxygen-driven nebulisation is unavoidable, $\mathrm{PtCO}_{2}$ is monitored if possible. 


\section{Additional files}

Additional file 1: Original Protocol. (DOC 209 kb)

Additional file 2: Protocol Version 2.0. (PDF $157 \mathrm{~kb}$ )

Additional file 3: Online supplement - Table S1. PtCO change $\geq 4 \mathrm{mmHg}$ according to randomised treatment. Table $\mathbf{S 2}$. $\mathrm{SpO}_{2}$ mixed linear model comparisons: Oxygen minus Air. Table S3. Heart Rate mixed linear model comparisons: Oxygen minus Air. (DOC 80 kb)

\section{Abbreviations}

AECOPD: Acute exacerbation of chronic obstructive pulmonary disease: BTS: British thoracic society; COPD: Chronic obstructive pulmonary disease; $\mathrm{FEV}_{1}$ : Forced expiratory volume over $1 \mathrm{~s}$; FVC: Forced vital capacity; MRINZ: Medical research institute of new zealand; $\mathrm{PaCO}_{2}$ : Partial pressure of arterial carbon dioxide; $\mathrm{PcapCO}_{2}$ : Partial pressure of capillary carbon dioxide PIS: Participant information sheet; $\mathrm{PtCO}_{2}$ : Partial pressure of transcutaneous carbon dioxide; SD: Standard deviation; Sentec: Transcutaneous monitor brand; $\mathrm{SpO}_{2}$ : Oxygen saturation measured by oximetry; $\mathrm{StO}_{2}$ : Oxygen saturation measured by transcutaneous monitor

\section{Acknowledgements}

We would like to give special thanks to all of the participants for their involvement in our study.

\section{Presentation of findings}

The preliminary results of this study were submitted as an abstract to the ERS 2017 Congress, and presented as a poster [18].

\section{Funding}

This study was funded by the Health Research Council of New Zealand. The funder of the study had no role in the study design, data collection, data analysis, data interpretation, or writing of the manuscript. The corresponding author had full access to all the data in the study and had final responsibility for the decision to submit for publication.

\section{Availability of data and materials}

The datasets used and/or analysed during the current study are available from the corresponding author on reasonable request.

\section{Authors' contributions}

RB was the principal investigator for the study, is guarantor for the study, and affirms that this manuscript is an honest, accurate, and transparent account of the study being reported; that no important aspects of the study have been omitted; and that any discrepancies from the study as planned (and, if relevant, registered) have been explained. GB, JP, SM and JB were investigators on the study and collected the data. MW performed the statistical analysis. GB wrote the first draft of the manuscript. RB and PS conceived the study and wrote the first draft of the protocol with JP. GB, JP, SM, PS, JB, JF, MW and RB all contributed to study design, interpretation of results, manuscript writing, and reviewed the final manuscript prior to submission. All authors had full access to all of the data (including statistical reports and tables) in the study and can take responsibility for the integrity of the data and the accuracy of the data analysis. No writing assistance was received. All authors read and approved the final manuscript.

\section{Ethics approval and consent to participate}

Ethics approval was obtained from the Health and Disability Ethics Committee, New Zealand (Reference 14/NTB/200). Written informed consent was obtained before any study-specific procedures.

\section{Consent for publication}

Not applicable.

\section{Competing interests}

All authors have completed the ICMJE uniform disclosure form at www.icmje.org/coi_disclosure.pdf. All authors have no competing interests to declare, other than the MRINZ receiving research funding from Health Research Council of New Zealand.

\section{Publisher's Note}

Springer Nature remains neutral with regard to jurisdictional claims in published maps and institutional affiliations.

\section{Author details}

${ }^{1}$ Capital and Coast District Health Board, Wellington, New Zealand. ${ }^{2}$ Medical Research Institute of New Zealand, Box 7902, Wellington, PO 6242, New Zealand. ${ }^{3}$ Victoria University Wellington, Wellington, New Zealand. ${ }^{4}$ Wellington School of Medicine \& Health Sciences, University of Otago Wellington, Wellington, New Zealand.

Received: 12 April 2018 Accepted: 10 September 2018

Published online: 03 October 2018

\section{References}

1. $M$ a A, Wills $K E$, Blizzard L, et al. Effect of high flow oxygen on mortality in chronic obstructive pulmonary disease patients in prehospital setting: randomised controlled trial. BMJ. 2010;341:C5462.

2. Murphy R, Driscoll P, O'Driscoll R. Emergency oxygen therapy for the COPD patient. Emerg Med J. 2001;18:333-9.

3. Edwards $L$, Perrin $K$, Williams $M$, et al. Randomised controlled crossover trial of the effect on PtCO2 of oxygen-driven versus air-driven nebulisers in severe chronic obstructive pulmonary disease. Emerg Med J. 2012;29:894-8.

4. Gunawardena KA, Patel B, Campbell IA, et al. Oxygen as a driving gas for nebulisers: safe or dangerous? Br Med J (Clin Res Ed). 1984;288:272-4.

5. O'Donnell D, Kelly CP, Cotter P, Clancy L. Use of oxygen driven nebuliser delivery systems for beta-2 agonists in chronic bronchitis. Ir J Med Sci. 1985; 154:198-200.

6. British Thoracic Society Emergency Oxygen Guideline Group. BTS guidelines for oxygen use in adults in healthcare and emergency settings. Br Thorac Soc. 2017:72:1-214.

7. Wijesinghe $M$, Williams $M$, Perrin $K$, et al. The effect of supplemental oxygen on hypercapnia in subjects with obesity-associated hypoventilation: a randomised, crossover, clinical study. Chest. 2011;139:1018-24.

8. Perrin K, Wijesinghe M, Healy B, et al. Randomised controlled trial of high concentration versus titrated oxygen therapy in severe exacerbations of asthma. Thorax. 2011:66:937-41.

9. Rudolf $\mathrm{M}$, Turner JA, Harrison $\mathrm{BD}$, et al. Changes in arterial blood gases during and after a period of oxygen breathing in patients with chronic hypercapnic respiratory failure and in patients with asthma. Clin Sci. 1979; 57:389-96.

10. Senn O, Clarenbach CF, Kaplan V, et al. Monitoring carbon dioxide tension and arterial oxygen saturation by a single earlobe sensor in patients with critical illness or sleep apnea. Chest. 2005;128:1291-6.

11. Rodriguez P, Lellouche F, Aboab J, et al. Transcutaneous arterial carbon dioxide pressure monitoring in critically ill adult patients. Intensive Care Med. 2006:32:309-12.

12. McVicar J, Eager R. Validation study of a transcutaneous carbon dioxide monitor in patients in the emergency department. Emerg Med J. 2009;26:344-6.

13. Aubier M, Murciano D, Milic-Emili J, et al. Effects of the administartion of $\mathrm{O} 2$ on ventilation and blood gases in patients with chronic obstructive pulmonary disease during acute respiratory failure. Am Rev Resp Dis. 1980; 122:747-54.

14. Robinson TD, Freiberg DB, Regnis JA, Young $\amalg$. The role of hypoventilation and ventilation-perfusion redistribution in oxygen-induced hypercapnia during acute exacerbations of chronic obstructive pulmonary disease. Am J Respir Crit Care Med. 2000;161:1524-9.

15. van Geffen WH, Douma WR, Siebos DJ, Kerstjens HA. Bronchodilators delivered by nebuliser versus pMDI with spacer or DPI for eaxcerbations of COPD. Cochrane Database Syst Rev 2016 (8): CD011826, Epub 2016 Aug 29.

16. Auerbach D, Hill C, Baughman R, et al. Routine nebulised ipratropium and albuterol together are better than either alone in COPD. Chest. 1997;112:1514-21.

17. Kane B, Turkington PM, Howard LS, et al. Rebound hypoxaemia after administration of oxygen in an acute exacerbation of chronic obstructive pulmonary disease. BMJ. 2011;342:d1557.

18. Bardsley G, McKinstry S, Pilcher J, et al. Oxygen compared to air driven nebulisers for acute exacerbations of COPD: a randomised controlled trial. ERJ. 2017:50:PA684. 